\title{
A giant dermoid cyst of the orbit present in adulthood - a case report
}

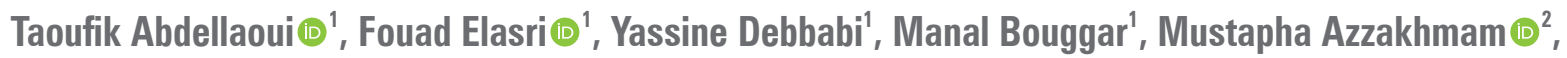 \\ Rachid Zerrouk', Yassine Mouzari', Karim Reda', Abdelbarre Oubaaz ${ }^{1}$ \\ 'Department of Ophthalmology, Military Teaching Hospital Med-V, Rabat, Morocco \\ ${ }^{2}$ Department of Pathology, Military Teaching Hospital Med-V, Rabat, Morocco
}

\begin{abstract}
Dermoid cysts are common orbital developmental tumors in childhood. They account for 3 to $9 \%$ of primary orbital tumors. They are considered to be congenital, but not all of them are diagnosed at birth. Dermoid cysts are benign choristomas that arise from sequestration of ectoderm along the lines of embryonic fusion of mesodermal processes destined to be bone. In most cases, the lesion is close to the zygomatic-frontal suture leading to a superotemporal subcutaneous mass in the eyebrow or eyelid. The intraorbital location is relatively rare and more prone to complications, particularly compressive. We report the case of a 41-year-old man with a large intraorbital tumor. The imaging aspects were evocative of an extraconal dermoid cyst. The tumor was resected through a transconjunctival route. Histopathologic examination confirmed the diagnosis of dermoid cyst.
\end{abstract}

KEY WORDS: dermoid; cyst; orbit; MRI; orbitotomy; proptosis

Ophthalmol J 2021; Vol. 6, 53-56

\section{INTRODUCTION}

Dermoid cysts are benign developmental tumors lined by squamous epithelium with dermal elements such as hair follicles, sebaceous, and sweat glands. Their lumens are filled with keratin, hair, smooth muscle, and lipid debris. The majority of dermoid cysts are superficial and present in early childhood as swelling in the eyebrow or eyelid. Deep lesions are rare, more insidious, presenting in the teenage years and beyond. The term "giant orbital dermoid" has been used to describe an orbital cyst equal in size to or larger than the affected eyeball [1]. In this report, the authors describe a case that presented in adulthood.

\section{CASE PRESENTATION}

A 41-year-old man presented with progressive proptosis of the left eye, which he had been having for ten years, without a decrease of vision, pain or diplopia. Since his vision was correct, the patient was not too embarrassed by his appearance, which delayed the consultation. His visual acuity was $6 / 6$ in both eyes. On inspection, the left eye showed non-axial proptosis with inferolateral ocular displacement (hypoglobus) (Fig. 1A). On palpation, orbital margins were intact, and there was no palpable mass.

Results of the pupillary examination were normal. Ocular motility showed a mild decrease in the 

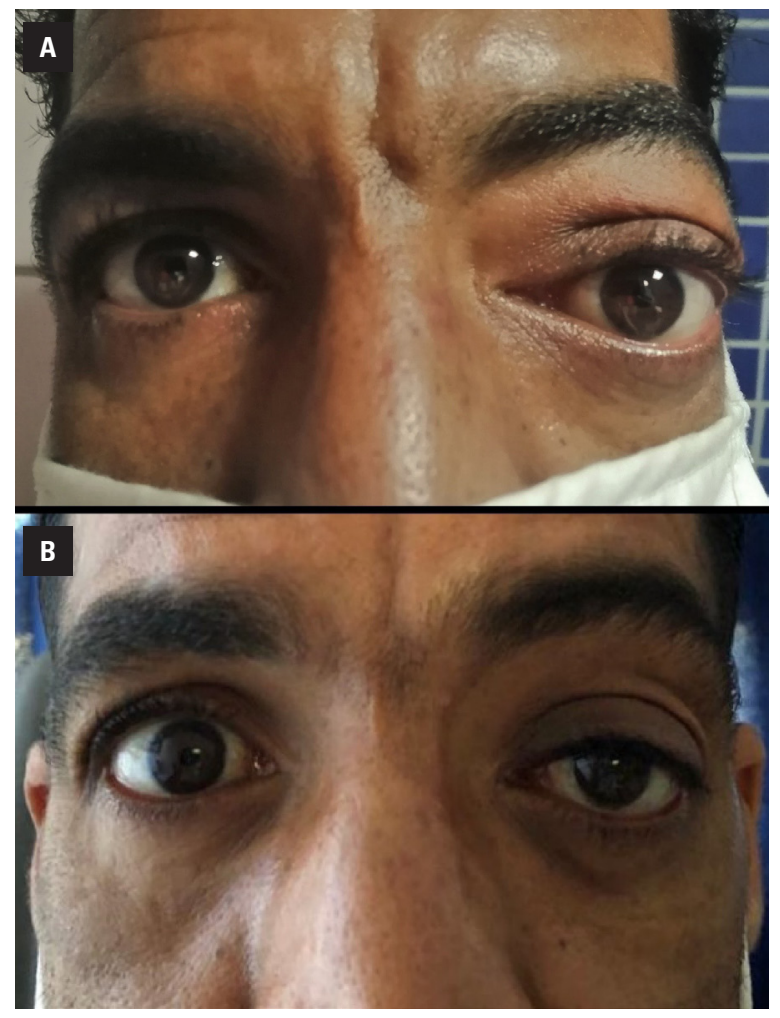

FIGURE 1. A. Frontal photograph of the patient at presentation: proptosis with inferolateral ocular displacement (hypoglobus) of the left eye. B. One-week postoperative, complete resolution of the proptosis and hypoglobus

abduction of the left eye. Anterior and posterior segment examination was unremarkable.

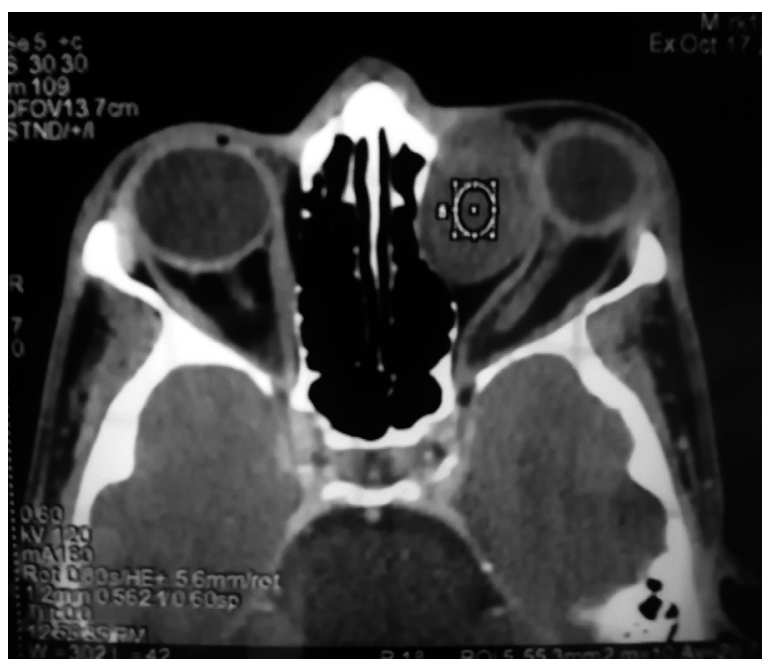

FIGURE 2. Computed tomography (CT) revealed a heterogeneous, mixed solid and fat density lesion, measuring $33 \times 28 \mathrm{~mm}$, enlarging the left orbit, superior and medial to the left globe

Computed tomography (CT) revealed a heterogeneous, mixed solid and fat density tumor, measuring $33 \times 28 \mathrm{~mm}$, enlarging the orbit, superior and medial to the left globe (Fig. 2). MRI showed a well-defined bilobular mass measuring $30 \times 30 \times 26 \mathrm{~cm}$ with two components: fat on top (Hypersignal T2, which showed suppression with fat suppression images) and mucinous in the lower part (hypersignal T1 persisting with fat-suppression images). Enhancement was minimal (Fig. 3). These

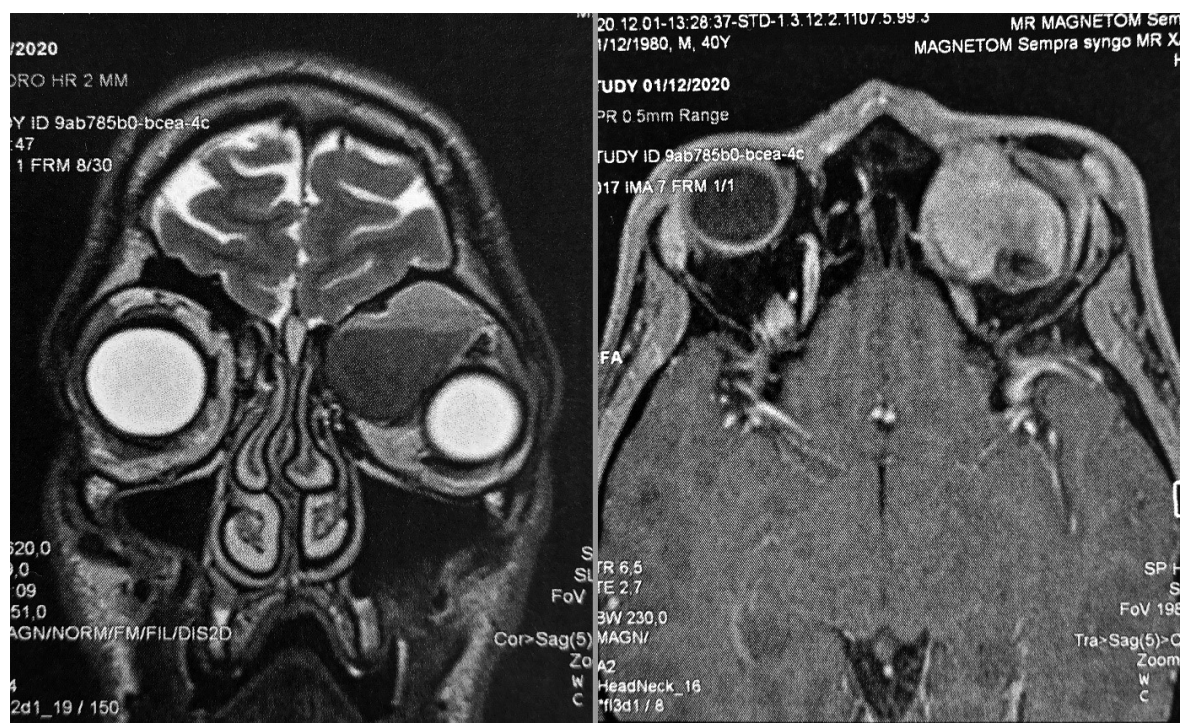

FIGURE 3. MRI images: well-defined, bilobular mass measuring $30 \times 30 \times 26 \mathrm{~cm}$ with two components: fat on top (hypersignal T2, which showed suppression with fat suppression images) and mucinous in the lower part (hypersignal T1 persisting with fat-suppression images) 
clinical and imaging findings were consistent with a dermoid cyst of the orbit.

The patient underwent surgical excision of the lesion via the anterior transconjunctival route. The lesion was very adherent to the adjacent structures. Given the impossibility of monobloc removal, it was decided to empty the cyst completely before proceeding with extracapsular dissection. After removing the whole cyst contents was done, great care was taken to remove the entire capsule intact. Results of histopathologic examination confirmed the diagnosis of a dermoid cyst. Postoperatively, the proptosis and hypoglobus resolved utterly (Fig. 1B).

\section{DISCUSSION}

Dermoid cysts are dysembryoplastic congenital tumors of ectodermal origin. They are a subset of benign heterotopic neoplasms termed choristomas (a developmental tumor-like growth of microscopically normal tissue in an abnormal location). The pathogenesis is not clearly established. They arise between $3^{\text {th }}$ and $5^{\text {th }}$-week gestation [2] from ectodermal rests "pinched off" at suture lines [3]. This could explain why intraorbital dermoid cysts emerging from the orbital bone walls develop outside the muscular cone. This tissue fragment (choristoma) continues its growth autonomously. It can manifest in childhood, puberty, or later in life [4]. Histologically, they are encapsulated by surface epithelium resembling epidermis and dermis, containing sebaceous glands, sweat glands, and hair follicles. The inner lumen may contain collagen, keratin, fat, sebaceous fluid, hair shafts, cholesterol, and calcium $[5,6]$.

Dermoid cysts are common lesions of the orbital region. They are cystic tumors that differ from solid dermoids that occur in the conjunctiva and cornea. Orbital dermoid make up 3-9\% of all orbital masses [5]. A distinction is made between superficial and deep tumors, depending on their relationship to the orbital septum. The majority of dermoid cysts seen in ophthalmic practice are superficial and present in early childhood as soft tissue swelling in the eyebrow or eyelid. Deeper lesions are rare and can reach large sizes because they grow indolently. They present later in life and require more extensive surgery [3].

Clinical manifestations are variable and depend on the location in the orbit. Superficial forms usually manifest as a subcutaneous palpebral swelling of the superolateral location, deforming the eye- brow. The tumor is painless, non-inflammatory, and easily palpable at the orbital rim. These preseptal superficial forms are usually expressed in childhood [7]. The deep, retro- or peribulbar forms are expressed as a slowly progressive, painless, irreducible, and nonaxial exophthalmitis. Deeper forms, contiguous to the optic nerve, can induce a decrease in visual acuity.

Sometimes there may be tearing secondary to compression or irritation of the lacrimal nerve, decreased visual acuity due to acquired hyperopia, or diplopia due to blockage of extra-ocular muscles. The acute aggravation of exophthalmitis, the sudden appearance of ophthalmoplegia, pain, or inflammatory signs may indicate the rupture of the cyst or intracystic hemorrhage. Sometimes the cyst is revealed by cutaneous or conjunctival fistulization $[7,8]$.

CT-scan allows specifying the location and density of the tumor, its relationship to adjacent structures. The dermoid cyst has characteristic features. It is typically well-defined and has an enhancing wall and a non-enhancing lumen with irregular dense areas inside corresponding to epithelial debris. The presence of calcifications around the lesion would be an evocative sign $[6,8]$. The MRI has a definite interest in evaluating the cyst's relationship with the muscles, periorbita, and optic nerve. On MRI, the lesion appears as a well-defined mass. The intensity of the signal depends on the material contained in the cyst lumen. If the content is lipidic, it appears hyperintense on $\mathrm{T} 1$-weighted imaging. If the cyst contains higher levels of protein, it appears hyperintense on both T1- and T2-imaging. Enhancement is minimal since the cyst does not have blood vessels $[6,9]$.

Even if they are benign, surgical resection is the rule because the local and locoregional evolution of these cysts can be marked by complications related to the increase in tumor volume and its mass effect leading to exophthalmos oculomotor paralysis or a decrease in visual acuity. A cyst itself can cause the complications such as recurrent local inflammatory flare-ups emerging due to the cyst's rupture [10]. Externalization of the cyst contents is possible the following fistulization to the skin or migration of the oil contents along the muscle sheaths resulting in the appearance of sub-conjunctival lipid droplets [11].

The treatment is only surgical and consists of removing the cyst by insisting on its extensions to avoid recurrence since the germinative center is in 
the wall of the cyst. The dermoid cyst content (lipid and keratin) induces inflammation in the cyst wall and secondary fibrosis, which may complicate surgery [4]. The surgical approach can be anterior transconjunctival, lateral, medial, or transcranial orbitotomy, considering the size of the tumor, its topography, and its extension. Whatever the surgical approach, it is imperative that the excision be complete to avoid recurrences [12]. Sometimes when the tumor is very large, the surgeon may aspirate its contents to facilitate its excision. The medial orbitotomy is used in the case of an internal tumor. Lateral orbitotomy may be more practical in the case of large posterior cysts. The neurosurgical approach, which consists of a subfrontal route after frontal or frontotemporal craniotomy, allows excellent access to the entire orbital cavity and remains a suitable approach in very large tumors and in the interest of a monobloc resection [8]. The anterior transconjunctival orbitotomy is of interest mainly in anterior lesions located anterior to the globe's equator with better esthetic results and lower hemorrhagic risk. In the case we present, this approach was used despite a rather posterior localization of the tumor because it was directly accessible.

\section{CONCLUSION}

Given their location and despite being histologically benign, these tumors, when they are long-neglected, can induce complications on adjacent structures. Fortunately for our patient, the damage was only aesthetic. A right choice of the surgical approach and a meticulous dissection often allow a complete excision to prevent recurrence.

\section{Disclosure of interest}

The Authors declare that they have no conflict of interest.

\section{Funding}

None.

\section{REFERENCES}

1. Mukherjee $B$, Desai A. A giant dermoid cyst of the orbit. Orbit. 2019; 38(2): 158-161, doi: 10.1080/01676830.2018.1457060, indexed in Pubmed: 29589985.

2. Pollard ZF, Harley RD, Calhoun J. Dermoid cysts in children. Pediatrics. 1976; 57(3): 379-382, indexed in Pubmed: 1256948.

3. Lane CM, Ehrlich WW, Wright JE. Orbital dermoid cyst. Eye (Lond). 1987; 1 (Pt 4): 504-511, doi: 10.1038/eye.1987.76, indexed in Pubmed: 3443205.

4. Abou-Rayyah $\mathrm{Y}$, Rose GE, Konrad H, et al. Clinical, radiological and pathological examination of periocular dermoid cysts: evidence of inflammation from an early age. Eye (Lond). 2002; 16(5): 507-512, doi: 10.1038/sj.eye.6700045, indexed in Pubmed: 12194059.

5. Sherman RP, Rootman J, Lapointe JS. Orbital dermoids: clinical presentation and management. Br J Ophthalmol. 1984; 68(9): 642-652, doi: 10.1136/bjo.68.9.642, indexed in Pubmed: 6466593.

6. Shields JA, Shields CL. Orbital cysts of childhood--classification, clinical features, and management. Surv Ophthalmol. 2004; 49(3): 281-299, doi: 10.1016/j.survophthal.2004.02.001, indexed in Pubmed: 15110666.

7. Civit T, Joud A, Klein O. [Congenital orbital tumors in adults (dermoid cysts)]. Neurochirurgie. 2010; 56(2-3): 183-186, doi: 10.1016/j. neuchi.2010.02.019, indexed in Pubmed: 20303551.

8. Taha $S$, Doe K, Compeyre $S$, et al. [Intraorbital dermoid cyst: case report]. Neurochirurgie. 2009; 55(6): 577-580, doi: 10.1016/j.neuchi.2008.12.002, indexed in Pubmed: 19368946.

9. Pham NSi, Dublin AB, Strong EB. Dermoid cyst of the orbit and frontal sinus: a case report. Skull Base. 2010; 20(4): 275-278, doi: 10.1055/s0030-1247631, indexed in Pubmed: 21311621.

10. Gotzamanis A, Desphieux JL, Pluot M, et al. [Dermoid cysts. Epidemiology, clinical and anatomo-pathologic aspects, therapeutic management] . J Fr Ophtalmol. 1999; 22(5): 549-553, indexed in Pubmed: 10417915.

11. Howard G, Nerad J, Bonavolonta G, et al. Orbital Dermoid Cysts Located within the Lateral Rectus Muscle. Ophthalmology. 1994; 101(4): 767-771, doi: 10.1016/s0161-6420(94)31268-1, indexed in Pubmed: 8152773.

12. Wang $\mathrm{Y}, \mathrm{Zhao} \mathrm{HP}$, Zhu H, et al. [Clinical manifestations, diagnosis and management of recurrent orbital dermoid cysts]. Zhonghua Yan Ke Za Zhi. 2010; 46(4): 295-298, indexed in Pubmed: 20654053. 